\title{
The anterior chamber of the eye is a transplantation site that supports and enables visualisation of beta cell development in mice
}

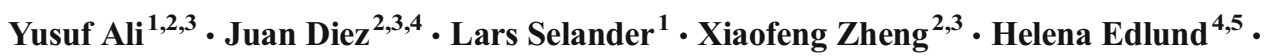 \\ Per-Olof Berggren ${ }^{1,2,3,4}$
}

Received: 2 August 2015 / Accepted: 15 January 2016 / Published online: 4 February 2016

(C) Springer-Verlag Berlin Heidelberg 2016

\begin{abstract}
Aims/hypothesis In vivo imaging of the developing pancreas is challenging due to the inaccessibility of the tissue. To circumvent this, on embryonic day 10.5 (E10.5) we transplanted a mouse developing pancreatic bud into the anterior chamber of the eye (ACE) to determine whether the eye is a useful transplant site to support pancreas development.

Methods We transplanted an E10.5 dorsal pancreatic bud into the ACE of a syngeneic recipient mouse. Using a mouse insulin promoter-green fluorescent protein (MIP-GFP) mouse as the tissue donor, we non-invasively imaged the pancreatic bud as it develops at single beta cell resolution across time. Results The transplanted pancreatic bud rapidly engrafts and vascularises when transplanted into the ACE. The pancreatic
\end{abstract}

Yusuf Ali, Juan Diez and Lars Selander contributed equally to this work.

Electronic supplementary material The online version of this article (doi:10.1007/s00125-016-3883-x) contains peer-reviewed but unedited supplementary material, which is available to authorised users.

Yusuf Ali

yusuf.ali@ntu.edu.sg

1 The Rolf Luft Research Center for Diabetes and Endocrinology, Karolinska Institutet, Karolinska University Hospital, Stockholm, Sweden

2 Lee Kong Chian School of Medicine, Nanyang Technological University, 59 Nanyang Drive, Singapore 636 921, Republic of Singapore

3 Singapore Eye Research Institute, The Academia, Singapore, Republic of Singapore

4 Diabetes Research Institute, University of Miami Leonard M. Miller School of Medicine, Miami, FL, USA

5 Umeå Center for Molecular Medicine, Umeå University, Umeå, Sweden progenitor cells differentiate into exocrine and endocrine cells, including cells expressing insulin, glucagon and somatostatin. The morphology of the transplanted pancreatic bud resembles that of the native developing pancreas. Beta cells within the transplanted pancreatic bud respond to glucose in a manner similar to that of native fetal beta cells and superior to that of in vitro developed beta cells. Unlike in vitro grown pancreatic explants, pancreatic tissue developing in the ACE is vascularised, providing the developing pancreatic tissue with a milieu resembling the native situation.

Conclusions/interpretation Altogether, we show that the ACE is able to support growth, differentiation and function of a developing pancreatic bud across time in vivo.

Keywords Beta cells · Eye transplantation · In vivo imaging · Pancreas development $\cdot$ Pancreatic islets

$\begin{array}{ll}\text { Abbreviations } \\ \text { ACE } & \text { Anterior chamber of the eye } \\ \text { E10.5 } & \text { Embryonic day 10.5 } \\ \text { MIP-GFP } & \begin{array}{l}\text { Mouse insulin promoter-green } \\ \text { fluorescent protein }\end{array} \\ \text { PDX1 } & \text { Pancreatic and duodenal homeobox 1 } \\ \text { PTD } & \text { Post-transplantation day }\end{array}$

\section{Introduction}

One of the most attractive therapeutic approaches to battling diabetes is to transplant functional islets into a patient. However, with scarcity of tissue donors, a possible source is to derive beta cells from either embryonic stem cells or induced pluripotent stem cells in vitro. Recent articles have reported reliable protocols to produce functional human beta cells 
in vitro $[1,2]$. However, these islets still lack bona fide complexity of intermingled heterogeneous islet cells that through paracrine interactions secrete key metabolic hormones in a highly tuned manner, thus falling short in recapitulating the functional complexities of islets. Our inability to visualise islets as they develop over time in vivo needs to be addressed so as to better understand this intricate process. A promising candidate site, not explored in islet development, is the anterior chamber of the eye (ACE) [3, 4]. Islets transplanted into the ACE maintain euglycaemia in streptozotocin-treated mouse models of diabetes, and in these mice hyperglycaemia only ensues upon enucleation (eye removal) [3].

In this article we show that the ACE is a useful transplantation site for supporting pancreas development in vivo. Furthermore, as the cornea is a natural body window for non-invasive, longitudinal, in vivo three-dimensional imaging, we are able to study beta cell differentiation and maturation in a living animal. Our approach combines powerful highresolution confocal microscopy with intraocular transplantation to enable non-invasive monitoring of the same individual pancreatic bud longitudinally with the potential for direct biological/pharmacological manipulation of the local environment in the ACE [4] to identify key regulatory mechanisms and factors involved in pancreatic islet development.

\section{Methods}

Mice, tissue preparation and ACE transplantation The animal experiments were approved by ethics committees at Karolinska Institutet (Stockholms Norra Försöksdjursetiska Nämnd). All mice were housed and bred in the animal facility at Karolinska Institutet at $22 \pm 2^{\circ} \mathrm{C}$, maintained on a $12 \mathrm{~h} / 12 \mathrm{~h}$ light/dark cycle and fed ad libitum. Mouse insulin promotergreen fluorescent protein (MIP-GFP) male mice (B6.Cg-Tg(Ins 1-EGFP)1Hara/J) on a C57BL/6-J background [5] were mated with C57BL/6-J females (The Jackson Laboratory, Bar Harbor, ME, USA) to generate 10.5 day embryos (E10.5), from which dorsal pancreatic buds were mechanically dissected using etched tungsten (Goodfellow Cambridge, Huntingdon, UK) tips. Buds that were damaged following mechanical dissection were excluded from the study. 8- to 10-week old C57BL/6-J male mice were used as recipients. For transplantation, the cornea was penetrated with a 27-gauge needle, and a thin glass capillary was used to transfer one pancreatic bud into the ACE of anaesthetised (2.5\% isoflurane; Baxter, Deerfield, IL, USA) recipient mice. A subcutaneous injection of Temgesic $(2 \mu \mathrm{g} / \mathrm{mouse}$; Schering-Plough, Kenilworth, NJ, USA) was administered for postoperative analgesia.

In vitro cultures Pancreatic buds isolated from E10.5 embryos were cultured on filters (EMD Millipore, Billerica, MA,
USA) in 24-well plates (Falcon; Fisher Scientific, Hampton, NH, USA) with $400 \mu$ of medium consisting of Dulbecco's modified Eagle's medium (Invitrogen, Carlsbad, CA, USA) supplemented with 10\% FCS (Invitrogen) as described previously [6]. Tissue was cultured for 10 days at $37^{\circ} \mathrm{C}$ and in $5 \%$ $\mathrm{CO}_{2}$, and medium was changed every other day.

Microscopy The in vivo imaging set-up was as previously described $[3,4]$. To visualise blood vessels in vivo, dextran labelled with Texas Red $(100 \mu \mathrm{l}$ of $10 \mathrm{mg} / \mathrm{ml}$; Molecular Probes, Eugene, OR, USA) was injected into the tail vein. TCS SP2 (Leica Microsystems, Wetzlar, Germany) and TCS SP5 (Leica Microsystems) confocal microscopes were used for both in vivo and immunofluorescence imaging. All antibodies were validated in-house prior to use. Primary antibodies used for immunofluorescence imaging were: guinea pig anti-insulin (1:200; Abcam, Cambridge, UK), rabbit antiglucagon (1:100; BioGenex, Fremont, CA, USA), mouse anti-glucagon (1:100, Sigma-Aldrich, St Louis, MO, USA), rabbit anti-somatostatin (1:100; Dako, Carpinteria, CA, USA), rat anti-CD31 (1:100; BD Biosciences, Franklin Lakes, NJ, USA), rabbit anti-amylase (1:100; SigmaAldrich), rabbit anti-GLUT2 (1:100; in house) and rabbit anti-pancreatic and duodenal homeobox 1 (PDX1) (1:100; in house). Secondary antibodies used were appropriate combinations of Alexa 405, 488, 546, 594 and 633 (1:500; Invitrogen).

Insulin secretion analysis ACE grafts were subjected to static incubations (30 $\mathrm{min}$ ) in buffered solution $(25 \mathrm{mmol} / 1 \mathrm{HEPES}$, $125 \mathrm{mmol} / 1 \mathrm{NaCl}, 5.9 \mathrm{mmol} / 1 \mathrm{KCl}, 1.28 \mathrm{mmol} / 1 \mathrm{CaCl}_{2}$, $1.2 \mathrm{mmol} / 1 \mathrm{MgCl}_{2}, 0.1 \% \mathrm{BSA}, \mathrm{pH} 7.4$ ) at different glucose concentrations, with or without forskolin $(10 \mu \mathrm{mol} / \mathrm{l})$, and with or without $\mathrm{KCl}(25 \mathrm{mmol} / \mathrm{l})$ (Sigma-Aldrich). Insulin in the media was measured by a two-photon fluorescence excitation microparticle fluorometry assay for insulin (ArcDia, Turku, Finland).

\section{Results}

The ACE supports pancreatic bud development To determine whether the ACE is able to support fetal pancreas growth, E10.5 dorsal pancreatic buds were dissected and immediately transplanted into the ACE of a syngeneic recipient. We progressively monitored changes in bud size over a period of 10 days, corresponding to maximum gestational time, with an objective placed above the cornea. The diameter of the pancreatic bud prior to transplantation (post-transplantation day [PTD]-0) was approximately $100 \mu \mathrm{m}$. The bud size doubled after 4 days (PTD-4), and by day 7 (PTD-7) the graft diameter was about $350 \pm 50 \mu \mathrm{m}$ (Fig. 1a, Bright field). Few GFP-positive cells from MIP-GFP pancreatic buds were visualised at PTD-4, but by PTD-7 we observed numerous 

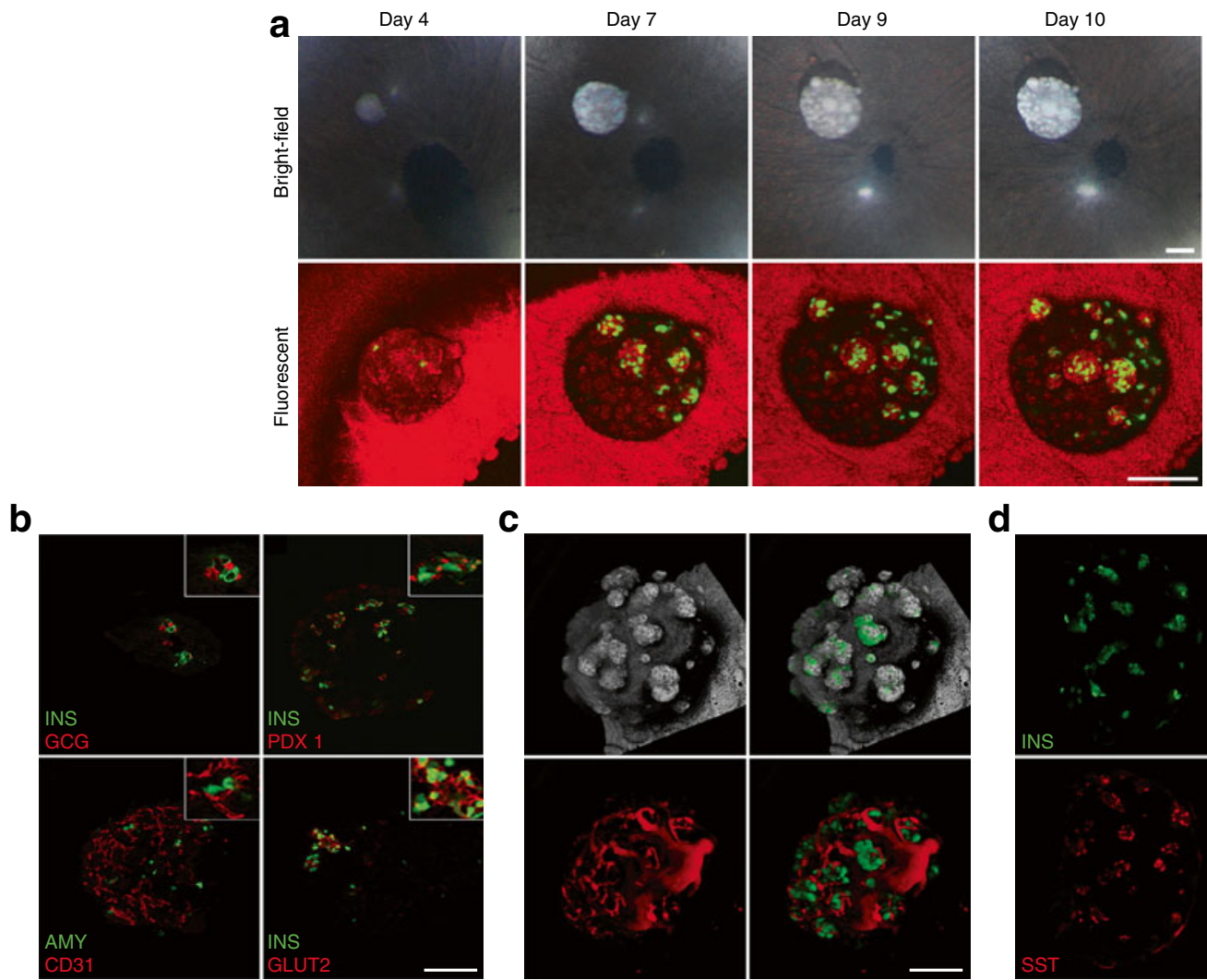

Fig. 1 E10.5 pancreatic buds transplanted into the ACE express most pancreatic cell markers. (a) Bright-field and fluorescent images of pancreatic buds across time. GFP-expressing cells (green) correspond to insulin-positive cells, and reflected light (red) represents both iris and pancreatic bud $(n=20)$. (b) Immunofluorescence images of pancreatic buds showing the expression of insulin (INS), glucagon (GCG) and PDX1 in cells that are forming small clusters; amylase (AMY)- and CD31-expressing cells show the presence of exocrine and endothelial cells, respectively; insulin with GLUT2 labels beta cells of transplanted pancreatic buds. Inserts show $\times 40$ magnification of small clusters. (c) Representative three-dimensional reconstruction of pancreatic bud in vivo on PTD-21.

GFP-positive cells organised in discrete cell clusters which further grew in size by PTD-10 (Fig. 1a, Fluorescent). We found prominent CD31-positive staining and amylasepositive cells at PTD-10, confirming the presence of vascular endothelial cells and exocrine cells, respectively (Fig. 1b). In fact, blood vessels can be seen penetrating the pancreatic bud with cell movement within the lumen, suggesting functional, intact blood vessels. Graft vascularisation increased dramatically until PTD-10, when tissue appeared fully vascularised at a level similar to that of native embryonic pancreas (electronic supplementary material [ESM] Fig. 1). We observed both GLUT2 and PDX1 presence in GFP-positive insulin cells which clustered with glucagon-positive cells within islet-like structures at PTD-10 (Fig. 1b). There was no observed colocalisation of insulin and glucagon at the cellular level, suggesting the absence of dual-hormone-expressing cells at PTD-10 (Fig. 1b). The pancreatic tissue at PTD-21 did not show any morphological change, including full

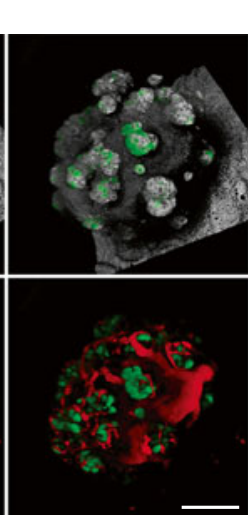

d

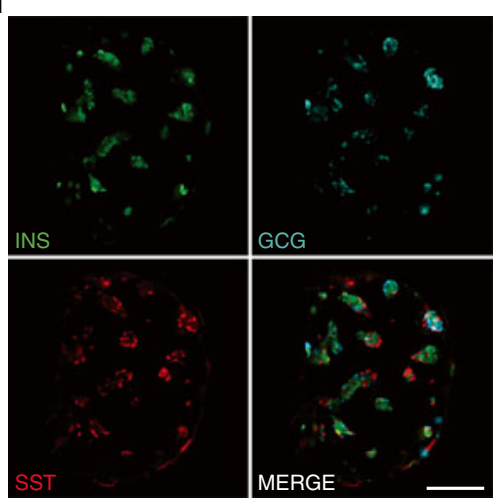

Reflected light (grey) shows the surface of the transplanted pancreatic bud and iris, and GFP (green) corresponds to insulin-expressing cell clusters. The blood vessel network (red) is visualised by injection of Texas Redlabelled dextran into the tail vein of the host animal, showing that the pancreatic tissue is highly vascularised $(n=5)$. (d) The isolated engrafted tissue was subsequently subjected to wholemount immunohistochemistry, and confocal sections were scanned and presented as maximum projections. The three examined pancreatic endocrine cell types could all be found in organised clusters and include insulin (green), glucagon (cyan) and somatostatin (SST; red). A merged image of all three stains shows the endocrine clusters $(n=6)$. Scale bars represent $200 \mu \mathrm{m}$

vascularisation, as a consequence of the prolonged incubation time, when compared with PTD-10 grafts. Subsequent in vivo imaging at PTD-21 showed that the graft was well vascularised, with the areas of GFP-positive cell clusters being particularly dense with fine blood vessels, similar to islets of the pancreas (Fig. 1c). Immunostaining on PTD-21 explants showed that clusters of insulin-expressing GFPpositive cells also contained bona fide islet cell types such as glucagon- and somatostatin-positive cells (Fig. 1d). In addition, we compared exocrine and endocrine differentiation in embryonic tissue and in the E10.5 dorsal buds maintained in culture or engrafted in the ACE for 4 and 7 days. Amylase expression in ACE grafts and in cultured explants was similar and lower when compared with the expression in the embryonic pancreas in situ (ESM Fig. 2a). Furthermore, the alpha cell to beta cell ratio was similar in all cases (eye grafts, explants or in situ) after 7 days (or E17.5) (ESM Fig. 2b). 
Insulin secretion was induced in ACE-transplanted buds but not in in vitro cultured buds To determine functionality, ACE-transplanted buds were removed at PTD-10 and exposed to a series of beta cell stimuli. In parallel, we performed the same assay on pancreatic buds cultured in vitro for 10 days. As forskolin has been shown to stimulate glucose responsiveness in immature beta cells [7], we co-incubated buds with forskolin $(10 \mu \mathrm{mol} / \mathrm{l})$ and glucose $(11 \mathrm{mmol} / \mathrm{l})$ and found enhanced insulin secretion only from ACE-transplanted buds (tenfold) and not from in vitro cultured buds (Fig. 2, ESM Fig. 3). None of forskolin alone, high glucose (11 mmol/l) alone or prolonged transplant incubation within the ACE led to enhanced insulin secretion from these buds, corroborating an earlier observed characteristic of fetal beta cells [8].

\section{Discussion}

So far our inability to visualise pancreatic islets as they develop in vivo has severely hampered our understanding of pancreatic islet development. Existing in vivo imaging approaches such as multiphoton microscopy require invasive surgical access to the pancreas and/or to other transplantation sites; and MRI, positron emission tomography and bioluminescence lack the spatial resolution to enable studies on islet cell development. We now demonstrate that pancreatic buds engrafted in the ACE develop similarly to the native pancreas, with cells expressing PDX1, somatostatin and glucagon. Moreover, developing islets in the ACE respond to both glucose and forskolin, similar to fetal islets [7]. Therefore, isolated E10.5 pancreatic buds transplanted into the $\mathrm{ACE}$

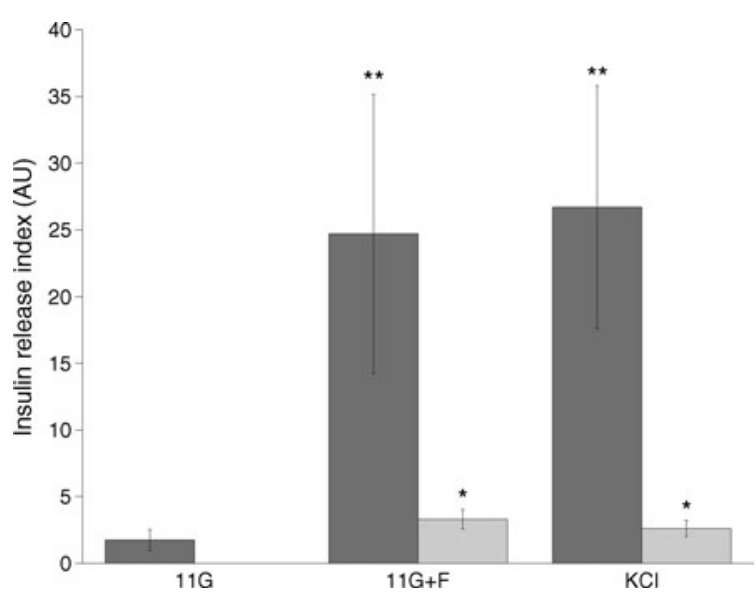

Fig. 2 Comparison of insulin secretion from ACE-transplanted buds and in vitro cultured buds. Relative insulin release from pancreatic buds extracted from the ACE after 10 days (dark grey bars) and pancreatic buds grown in vitro for 10 days (light grey bars). $11 \mathrm{G}$ refers to $11 \mathrm{mmol} / 1$ glucose, $\mathrm{F}$ represents $10 \mu \mathrm{mol} / \mathrm{l}$ forskolin, and $\mathrm{KCl}$ refers to exogenously added $25 \mathrm{mmol} / 1 \mathrm{KCl}$. Bars represent mean average \pm SEM normalised to secretion following $3 \mathrm{mmol} / \mathrm{l}$ glucose $(n=7$ and $n=8)$. Student's $t$ test was used for statistical analyses; $p$ values $<0.05(*)$ and $<0.01(* *)$, compared with respective $11 \mathrm{G}$ insulin secretion, represent significance recapitulate the development of the embryonic pancreas in terms of morphology, gene expression and function.

Our results suggest that at this developmental stage (E10.5), intrinsic signals within the pancreatic bud are sufficient to drive differentiation of pancreas-specific cells. The differentiation process within the ACE may be facilitated by the rapid revascularisation of the graft, which seems to be completed by PTD-10. A three-dimensional reconstruction of these blood vessels shows that, similar to the native pancreas, there is a high density of fine blood vessels in the isletlike endocrine clusters of the pancreas which supply the islet cells with nutrients and growth factors necessary for islet development [9]. With appropriate reporter constructs and transgenic lines, this transplantation model provides a unique opportunity to study and identify factors regulating development of the endocrine pancreas in vivo, in real time. This, together with the possibility to manipulate the transplanted pancreatic tissue with great precision, makes this method a powerful experimental model system for developmental studies. The ability to study pancreatic islet development longitudinally in an environment resembling the native milieu may help identify mechanisms that induce maturation of the endocrine pancreas [10]. Therefore, the ACE is a superior transplant and imaging site for studying in vivo mouse islet development and embryonic function at single-cell resolution, longitudinally and in a non-invasive manner.

Acknowledgements We thank E. Ilegems and S. Jacob (The Rolf Luft Research Center for Diabetes and Endocrinology, Karolinska Institutet, Stockholm, Sweden) for their technical assistance and critical reading of the manuscript.

Funding YA was supported in part by the Agency for Science, Technology and Research (A*STAR), Singapore. In addition, this work was partly supported by a start-up grant from the Lee Kong Chian School of Medicine, Nanyang Technological University, Singapore (separately for YA and P-OB), the Singapore Ministry of Education Academic Research Fund (2014-T1-001-149 (for YA)), the Swedish Diabetes Association, funds of Karolinska Institutet, the Swedish Research Council, the Novo Nordisk Foundation, the Erling-Persson Family Foundation, the Strategic Research Programme in Diabetes at Karolinska Institutet, ERC-2013-AdG 338936-BetaImage, the Knut and Alice Wallenberg Foundation, Skandia Insurance Company Ltd, the Diabetes and Wellness Foundation, the Bert von Kantzow Foundation, Svenska Diabetesstiftelsen, the Stichting af Jochnick Foundation, and the Diabetes Research Institute Foundation.

Duality of interest P-OB is co-founder and CEO of Biocrine, an unlisted biotech company that is using the ACE technique as a research tool. $\mathrm{HE}$ is a co-founder, shareholder and consultant of the unlisted biotech company Betagenon $\mathrm{AB}$. The other authors declare that there is no duality of interest associated with their contribution to this manuscript.

Contribution statement YA, JD and LS contributed to the conception and design, acquisition of data, analysis and interpretation of data and drafting of the article. XZ contributed to the acquisition of data and critical revision of the article. HE and P-OB contributed to the conception and design, and drafting of the article. All authors approved the final version to be published. P-OB is the guarantor of this work. 


\section{References}

1. Rezania A, Bruin JE, Arora P et al (2014) Reversal of diabetes with insulin-producing cells derived in vitro from human pluripotent stem cells. Nat Biotechnol 32:1121-1133

2. Pagliuca FW, Millman JR, Gurtler M et al (2014) Generation of functional human pancreatic beta cells in vitro. Cell 159:428-439

3. Speier S, Nyquist $\mathrm{D}$, Cabrera $\mathrm{O}$ et al (2008) Noninvasive in vivo imaging of pancreatic islet cell biology. Nat Med 14:574-578

4. Speier S, Nyqvist D, Kohler M, Caicedo A, Leibiger IB, Berggren PO (2008) Noninvasive high-resolution in vivo imaging of cell biology in the anterior chamber of the mouse eye. Nat Protoc 3: $1278-1286$

5. Hara M, Wang X, Kawamura T et al (2003) Transgenic mice with green fluorescent protein-labeled pancreatic beta-cells. Am J Physiol Endocrinol Metab 284:E177-E183
6. Ostrom M, Loffler KA, Edfalk S et al (2008) Retinoic acid promotes the generation of pancreatic endocrine progenitor cells and their further differentiation into beta-cells. PLoS One 3:e2841

7. Rorsman P, Arkhammar P, Bokvist K et al (1989) Failure of glucose to elicit a normal secretory response in fetal pancreatic beta cells results from glucose insensitivity of the ATP-regulated $\mathrm{K}^{+}$channels. Proc Natl Acad Sci U S A 86:4505-4509

8. Otonkoski T, Knip M, Wong I, Simell O (1991) Lack of glucoseinduced functional maturation during long-term culture of human fetal islet cells. Life Sci 48:2157-2163

9. Cleaver O, Dor Y (2012) Vascular instruction of pancreas development. Development 139:2833-2843

10. Stolovich-Rain M, Enk J, Vikesa J et al (2015) Weaning triggers a maturation step of pancreatic beta cells. Dev Cell 32:535-545 